\title{
Cohomological Property of Vector Bundles on Biprojective Spaces
}

\author{
Francesco Malaspina and Chikashi Miyazaki \\ Politecnico di Torino \\ Corso Duca degli Abruzzi 24, 10129 Torino, Italy \\ e-mail: francesco.malaspina@polito.it \\ Department of Mathematics, Faculty of Education, Kumamoto University \\ Kurokami 2-40-1, Chuo-ku, Kumamoto 860-8555, Japan \\ e-mail: cmiyazak@educ.kumamoto-u.ac.jp
}

\begin{abstract}
This paper investigates the cohomological property of vector bundles on biprojective space. We will give a criterion for a vector bundle to be isomorphic to the tensor product of pullbacks of exterior products of differential sheaves.
\end{abstract}

\section{Introduction}

The purpose of this paper is to study the cohomological property of vector bundle towards Horrocks-type criteria and to characterize the tensor product of pullbacks of exterior products of differential sheaves on biprojective space. Horrocks Theorem says that an ACM vector bundle on the projective space is isomorphic to a direct sum of line bundles. There are some attempts to generalize to the biprojective space, that is, some splitting criteria for a vector bundle on $\mathbb{P}^{m} \times \mathbb{P}^{n}$ to be isomorphic to a direct sum of the form $\mathcal{O}_{\mathbb{P}^{m} \times \mathbb{P}^{n}}\left(\ell_{1}, \ell_{2}\right)$ in [2, 4, 6]. In particular in [4] it is used a Beilinson type spectral sequence and $m$-blocks collection while in [2, 6] it is used a notion of Castelnuovo-Mumford regularity and Koszul complexes (for a similar approach on Grassmannians see [1]). In this paper we will give a cohomological criterion for a vector bundle on $\mathbb{P}^{m} \times \mathbb{P}^{n}$ to have a direct summand of the form $\Omega_{\mathbb{p} m}^{p} \otimes \Omega_{\mathbb{p} n}^{q}$ using the second approach.

Let us describe our perspective on the condition for a vector bundle to have a specific direct summand. We will begin a proof of the Horrocks theorem through the Castelnuovo-Mumford regularity according to [2]. Let $E$ be an ACM vector bundle on $\mathbb{P}^{n}$. Assume that $E$ is $m$-regular but not $(m-1)$-regular, see [8] for the definition and basic properties for $m$-regular. Then we have a surjective map $\varphi: \mathcal{O}_{\mathbb{P} n}^{\oplus} \rightarrow E(m)$. Since $E$ is ACM, we have $\mathrm{H}^{n}(E(m-1-n)) \neq 0$, and $\mathrm{H}^{0}\left(E^{\vee}(-m)\right) \neq 0$ by Serre duality. Thus we have a nonzero map $\psi: E(m) \rightarrow \mathcal{O}_{\mathbb{P} n}$. Since $\psi \circ \varphi$ is nonzero, it splits. Hence $\mathcal{O}_{\mathbb{P} n}$ is a direct summand of $E(m)$.

Now we will proceed the next step on studying a Buchsbaum vector bundle on $\mathbb{P}^{n}$, that is, $\mathfrak{m H}_{*}^{i}\left(\left.E\right|_{L}\right)=0$ for any $r$-plane $L$ of $\mathbb{P}^{n}, 1 \leq i<r \leq n$, where $\mathfrak{m}=\oplus_{\ell \geq 1} \Gamma\left(\mathcal{O}_{\mathbb{P}^{n}}(\ell)\right)$. Instead of using the regularity we will make use of the Koszul complex. Before giving a sufficient condition

The first author is member of the GNSAGA group of INdAM. The second author was partially supported by INdAM(Italy) and JSPS Kakenhi(C) (26400048).

Mathematics Subject Classification. 14J60.

Keywords and Phrases. Horrocks criterion, Segre product, Buchsbaum vector bundle 
for a vector bundle to be a direct sum of vector bundles of the form $\Omega_{\mathbb{P}^{n}}^{i}(\ell)$, we will describe important facts concerning the structure of Buchsbaum vector bundle.

Proposition $1.1([3,5])$. Let $E$ be a Buchsbaum vector bundle on $\mathbb{P}^{n}$. Then $E$ is isomorphic to a direct sum of vector bundles of the form $\Omega_{\mathbb{P} n}^{i}(\ell)$.

Proposition $1.2\left(\left[9,\left(\right.\right.\right.$ I.3.10)). Let $E$ be a vector bundle on $\mathbb{P}^{n}$. Let us define $\mathfrak{S}=\{(i, \ell) \mid 1 \leq i \leq$ $\left.n-1, \ell \in \mathbb{Z}, \mathrm{H}^{i}(E(\ell)) \neq 0\right\}$. Suppose that $\mathfrak{S}$ satisfies the following condition: "For $(i, \ell),(j, m) \in$ $\mathfrak{S}$, if $i \geq j$, then $i+\ell+1 \neq j+m$ ". Then $E$ is Buchsbaum.

From these facts we have observed the relation between the Buchsbaum property and the differential sheaves in (1.1) and a cohomological characterization of Buchsbaum modules in (1.2). Then we will give a straightforward proof of a more or less known result which illustrates the relation between the vanishings of the intermediate cohomologies and the exterior products of differential sheaves. The following is a starting point of our main result, Theorem 2.1.

Proposition 1.3. Let $E$ be a vector bundle on $\mathbb{P}^{n}$ with $\mathrm{H}^{p}(E) \neq 0$, where $1 \leq p \leq n-1$. If a vector bundle $E$ has the following condition:

(a) $\mathrm{H}^{i}(E(p-i+1))=0$ for $1 \leq i \leq p$.

(b) $\mathrm{H}^{i}(E(p-i-1))=0$ for $p \leq i \leq n-1$,

then $E$ contains $\Omega_{\mathbb{P}^{n}}^{p}$ as a direct summand.

Proof. By an exact sequence arising from the Koszul complex:

$$
0 \rightarrow \mathcal{O}_{\mathbb{P}^{n}} \rightarrow \mathcal{O}_{\mathbb{P}^{n}}^{\oplus}(1) \rightarrow \cdots \rightarrow \mathcal{O}_{\mathbb{P}^{n}}^{\oplus}(p) \rightarrow \Omega_{\mathbb{P}^{n}}^{p \vee} \rightarrow 0,
$$

we have a surjective map $\varphi: \mathrm{H}^{0}\left(E \otimes \Omega_{\mathbb{P} n}^{p \vee}\right) \rightarrow \mathrm{H}^{p}(E)$ from the assumption $\mathrm{H}^{1}(E(p))=\cdots=$ $\mathrm{H}^{p}(E(1))=0$. By an exact sequence arising from the Koszul complex:

$$
0 \rightarrow \mathcal{O}_{\mathbb{P}^{n}}(-n-1) \rightarrow \mathcal{O}_{\mathbb{P}^{n}}^{\oplus}(-n) \rightarrow \cdots \rightarrow \mathcal{O}_{\mathbb{P}^{n}}^{\oplus}(-p-1) \rightarrow \Omega_{\mathbb{P}^{n}}^{p} \rightarrow 0,
$$

we have a surjective map $\psi: \mathrm{H}^{0}\left(E^{\vee} \otimes \Omega_{\mathbb{p} n}^{p}\right) \rightarrow \mathrm{H}^{n-p}\left(E^{\vee}(-n-1)\right)$ from the assumption $\mathrm{H}^{p}(E(-1))=\cdots=\mathrm{H}^{n-1}(E(p-n))=0$, that is, $\mathrm{H}^{1}\left(E^{\vee}(-p-1)\right)=\cdots=\mathrm{H}^{n-p}\left(E^{\vee}(-n)\right)=0$.

As in [2] we have a nonzero element $f \in \mathrm{H}^{0}\left(E \otimes \Omega_{\mathbb{p} n}^{p \vee}\right)$ such that $\varphi(f)=s(\neq 0) \in \mathrm{H}^{p}(E)$. Let us take an element $s^{*} \in \mathrm{H}^{n-p}\left(E^{\vee}(-n-1)\right)$ corresponding to $s \in \mathrm{H}^{m}(E)$, there is a nonzero element $g \in \mathrm{H}^{0}\left(E^{\vee} \otimes \Omega_{\mathbb{P} n}^{p}\right)$ such that $\psi(g)=s^{*}(\neq 0) \in \mathrm{H}^{n-p}\left(E^{\vee}(-n-1)\right)$. Then $f$ and $g$ are regarded as elements of $\operatorname{Hom}\left(\Omega_{\mathbb{P}^{n}}^{p}, E\right)$ and $\operatorname{Hom}\left(E, \Omega_{\mathbb{P}^{n}}^{p}\right)$ respectively. From a commutative diagram:

$$
\begin{aligned}
& \mathrm{H}^{0}\left(E \otimes \Omega_{\mathbb{P}^{n}}^{p \vee}\right) \otimes \mathrm{H}^{0}\left(E^{\vee} \otimes \Omega_{\mathbb{P}^{n}}^{p}\right) \rightarrow \mathrm{H}^{0}\left(\Omega_{\mathbb{P}^{n}}^{p \vee} \otimes \Omega_{\mathbb{P}^{n}}^{p}\right) \cong \mathrm{H}^{0}\left(\mathcal{O}_{\mathbb{P}^{n}}\right) \\
& \mathrm{H}^{p}(E) \otimes \mathrm{H}^{n-p}\left(E^{\vee}(-n-1)\right) \quad \rightarrow \quad \mathrm{H}^{n}\left(\mathcal{O}_{\mathbb{P}^{n}}(-n-1)\right),
\end{aligned}
$$

a natural map $\mathrm{H}^{0}\left(E \otimes \Omega_{\mathbb{P}^{n}}^{p \vee}\right) \otimes \mathrm{H}^{0}\left(E^{\vee} \otimes \Omega_{\mathbb{P}^{n}}^{p}\right) \rightarrow \mathrm{H}^{0}\left(\mathcal{O}_{\mathbb{P}^{n}}\right)$ yields that $g \circ f$ is an isomorphism, which implies $\Omega_{\mathbb{P} n}^{p}$ is a direct summand of $E$.

\section{Cohomological Criterion of Vector Bundles on Biprojective Space}

What condition is required for a vector bundle $E$ on $\mathbb{P}^{m} \times \mathbb{P}^{n}$ to have a direct summand of the form $\Omega_{\mathbb{P} m}^{p} \otimes \Omega_{\mathbb{P n}}^{q}$ ? Although the exterior products of differential sheaves are the indecomposable 
Buchsbaum vector bundles on $\mathbb{P}^{n}$, The Buchsbaum property of differential sheaves are more complicated on $\mathbb{P}^{m} \times \mathbb{P}^{n}$, see [7]. This section is devoted to an answer of cohomological criteria of differential sheaves from the viewpoint of (1.3). Compared with an important result of [4, (4.11)], our theorem obtained from an elementary way concludes an isomorphism to just one bundle directly.

Theorem 2.1. Let $E$ be a vector bundle on $\mathbb{P}^{m} \times \mathbb{P}^{n}$ with $\mathrm{H}^{p+q}(E) \neq 0$, where $1 \leq p \leq m-1$ and $1 \leq q \leq n-1$. If a vector bundle $E$ has the following condition:

(a) $\mathrm{H}^{i}(E(a, b))=0$ for $1 \leq i \leq p+q, 0 \leq a \leq p, 0 \leq b \leq q$ with $i+a+b=p+q+1$.

(b) $\mathrm{H}^{i}(E(a, b))=0$ for $p+q \leq i \leq m+n-1, p-m \leq a \leq 0, q-n \leq b \leq 0$ with $i+a+b=p+q-1$,

then $E$ contains $\Omega_{\mathbb{P}^{m}}^{p} \otimes \Omega_{\mathbb{P}^{n}}^{q}$ as a direct summand.

Proof. Let us consider the exact sequences arising from the Koszul complexes

$$
0 \rightarrow \Omega_{\mathbb{P}^{m}}^{p} \rightarrow \mathcal{O}_{\mathbb{P}^{m}}^{f_{p}}(-p) \rightarrow \mathcal{O}_{\mathbb{P}^{m}}^{f_{p-1}}(-p+1) \rightarrow \cdots \rightarrow \mathcal{O}_{\mathbb{P}^{m}}^{f_{1}}(-1) \rightarrow \mathcal{O}_{\mathbb{P}^{m}} \rightarrow 0
$$

and

$$
0 \rightarrow \Omega_{\mathbb{P}^{n}}^{q} \rightarrow \mathcal{O}_{\mathbb{P}^{n}}^{e_{q}}(-q) \rightarrow \mathcal{O}_{\mathbb{P}^{n}}^{e_{q-1}}(-q+1) \rightarrow \cdots \rightarrow \mathcal{O}_{\mathbb{P}^{n}}^{e_{1}}(-1) \rightarrow \mathcal{O}_{\mathbb{P}^{n}} \rightarrow 0,
$$

where $f_{i}=\left(\begin{array}{c}m \\ i\end{array}\right)$ and $e_{j}=\left(\begin{array}{c}n \\ j\end{array}\right)$. By gluing the pull back by $p_{2}: \mathbb{P}^{m} \times \mathbb{P}^{n} \rightarrow \mathbb{P}^{n}$ of the dual of (2) tensored by $E$ and the pull back by $p_{1}: \mathbb{P}^{m} \times \mathbb{P}^{n} \rightarrow \mathbb{P}^{m}$ of the dual of (1) tensored by $E \otimes p_{2}^{*} \Omega_{\mathbb{P}^{n}}^{q \vee}$ we obtain an exact sequence

$$
\begin{gathered}
0 \rightarrow E \rightarrow E(0,1)^{e_{1}} \rightarrow E(0,2)^{e_{2}} \rightarrow \cdots \rightarrow E(0, q)^{e_{q}} \rightarrow E(1,0)^{f_{1}} \otimes p_{2}^{*} \Omega_{\mathbb{P}^{n}}^{q \vee} \\
\rightarrow E(2,0)^{f_{2}} \otimes p_{2}^{*} \Omega_{\mathbb{P}^{n}}^{q \vee} \rightarrow \cdots \rightarrow E(p, 0)^{f_{p}} \otimes p_{2}^{*} \Omega_{\mathbb{P}^{n}}^{q \vee} \rightarrow E \otimes p_{1}^{*} \Omega_{\mathbb{P}^{m}}^{p \vee} \otimes p_{2}^{*} \Omega_{\mathbb{P}^{n}}^{q \vee} \rightarrow 0 .
\end{gathered}
$$

Notice that

$$
\Omega_{\mathbb{P} n}^{q \vee} \cong \Omega_{\mathbb{P}^{n}}^{n-q}(n+1)
$$

In order to have a surjective map

$$
\varphi: \mathrm{H}^{0}\left(E \otimes p_{1}^{*} \Omega_{\mathbb{P} m}^{p \vee} \otimes p_{2}^{*} \Omega_{\mathbb{P} n}^{q \vee}\right) \rightarrow \mathrm{H}^{p+q}(E),
$$

we will show

(c.1) $\mathrm{H}^{1}\left(E(p, n+1) \otimes p_{2}^{*} \Omega_{\mathbb{P}^{n}}^{n-q}\right)=\cdots=\mathrm{H}^{p}\left(E(1, n+1) \otimes p_{2}^{*} \Omega_{\mathbb{P}^{n}}^{n-q}\right)=0$.

(c.2) $\mathrm{H}^{p+1}(E(0, q))=\cdots=\mathrm{H}^{p+q}(E(0,1))=0$.

The asserion (c.2) follows from the assumption (a). Since $\mathrm{H}^{i}(E(p-i+1, q))=\mathrm{H}^{i+1}(E(p-i+1, q-$ $1))=\cdots=\mathrm{H}^{i+q}(E(p-i+1,0))=0, i=1, \cdots, p$, we see that $\mathrm{H}^{i}\left(E(p-i+1, n+1) \otimes p_{2}^{*} \Omega_{\mathbb{P}^{n}}^{n-q}\right)=0$ from the exact sequence

$$
0 \rightarrow \mathcal{O}_{\mathbb{P}^{n}} \rightarrow \mathcal{O}_{\mathbb{P}^{n}}^{e_{n}}(1) \rightarrow \cdots \rightarrow \mathcal{O}_{\mathbb{P}^{n}}^{e_{n-q+1}}(q) \rightarrow \Omega_{\mathbb{P}^{n}}^{n-q}(n+1) \rightarrow 0
$$

by pulling back to $p_{2}$ and tensored by $E(p-i+1,0)$. Thus we obtain (c.1).

Next, let us consider the exact sequences arising from the Koszul complexes

$$
0 \rightarrow \mathcal{O}_{\mathbb{P} m}(-m-1) \rightarrow \mathcal{O}_{\mathbb{P} m}^{f_{m}}(-m) \rightarrow \cdots \rightarrow \mathcal{O}_{\mathbb{P}^{m}}^{f_{p+1}}(-p-1) \rightarrow \Omega_{\mathbb{P} m}^{p} \rightarrow 0
$$


and

$$
0 \rightarrow \mathcal{O}_{\mathbb{P}^{n}}(-n-1) \rightarrow \mathcal{O}_{\mathbb{P}^{n}}^{e_{n}}(-n) \rightarrow \cdots \rightarrow \mathcal{O}_{\mathbb{P}^{n}}^{e_{q+1}}(-q-1) \rightarrow \Omega_{\mathbb{P}^{n}}^{q} \rightarrow 0
$$

By gluing the pull back by $p_{2}$ of (4) tensored by $E^{\vee}(-m-1,0)$ and the pull back by $p_{1}$ of (3) tensored by $E^{\vee} \otimes p_{2}^{*} \Omega_{\mathbb{P} n}^{q}$ we obtain an exact sequence

$$
\begin{gathered}
0 \rightarrow E^{\vee}(-m-1,-n-1) \rightarrow E^{\vee}(-m-1,-n)^{e_{m}} \rightarrow \cdots \\
\cdots \rightarrow E^{\vee}(-m-1,-q-1)^{e_{p+1}} \rightarrow E^{\vee}(-m, 0)^{f_{m}} \otimes p_{2}^{*} \Omega_{\mathbb{P}^{n}}^{q} \rightarrow \cdots \\
\cdots \rightarrow E^{\vee}(-p-1,0)^{f_{p+1}} \otimes p_{2}^{*} \Omega_{\mathbb{P}^{n}}^{q} \rightarrow E^{\vee} \otimes p_{1}^{*} \Omega_{\mathbb{P} m}^{p} \otimes p_{2}^{*} \Omega_{\mathbb{P} n}^{q} \rightarrow 0 .
\end{gathered}
$$

In order to have a surjective map

$$
\psi: \mathrm{H}^{0}\left(E^{\vee} \otimes p_{1}^{*} \Omega_{\mathbb{P}^{m}}^{p} \otimes p_{2}^{*} \Omega_{\mathbb{P}^{n}}^{q}\right) \rightarrow \mathrm{H}^{m+n-p-q}\left(E^{\vee}(-m-1,-n-1)\right)
$$

we will show that $\mathrm{H}^{1}\left(E^{\vee}(-p-1,0) \otimes p_{2}^{*} \Omega_{\mathbb{P}^{n}}^{q}\right)=\cdots=\mathrm{H}^{m-p}\left(E^{\vee}(-m, 0) \otimes p_{2}^{*} \Omega_{\mathbb{P}^{n}}^{q}\right)=0$ and $\mathrm{H}^{m-p+1}\left(E^{\vee}(-m-1,-q-1)\right)=\cdots=\mathrm{H}^{m+n-p-q}\left(E^{\vee}(-m-1,-n)=0\right.$. By Serre duality, we have only to show

(d.1) $\mathrm{H}^{m+n-1}\left(E(p-m, 0) \otimes p_{2}^{*} \Omega_{\mathbb{P}^{n}}^{n-q}\right)=\cdots=\mathrm{H}^{n+p}\left(E(-1,0) \otimes p_{2}^{*} \Omega_{\mathbb{P}^{n}}^{n-q}\right)=0$.

(d.2) $\mathrm{H}^{n+p-1}(E(0,-n+q))=\cdots=\mathrm{H}^{p+q}(E(0,-1))=0$.

The assertion (d.2) follows from the assumption (b). Since $\mathrm{H}^{i}(E(n+p-i-1,-n+q))=\cdots=$ $\mathrm{H}^{i-n+q}(E(n+p-i-1,0))=0, i=n+p, \cdots, m+n-1$, from the assumption $(b)$, we see that $\mathrm{H}^{i}\left(E(n+p-i-1,0) \otimes p_{2}^{*} \Omega_{\mathbb{P}^{n}}^{n-q}\right)=0$ from the exact sequence

$$
0 \rightarrow \Omega_{\mathbb{P}^{n}}^{n-q} \rightarrow \mathcal{O}_{\mathbb{P}^{n}}^{e_{n-q}}(-n+q) \rightarrow \cdots \rightarrow \mathcal{O}_{\mathbb{P}^{n}}^{e_{1}}(-1) \rightarrow \mathcal{O}_{\mathbb{P}^{n}} \rightarrow 0
$$

by pulling back to $p_{2}$ and tensored by $E(n+p-i-1,0)$. Thus we obtain (d.1).

As in (1.3), for a nonzero element $s \in \mathrm{H}^{p+q}(E)$ and the corresponding element $s^{*} \in$ $\mathrm{H}^{m+n-p-q}\left(E^{\vee}(-m-1,-n-1)\right)$ by Serre duality, there are $f \in \mathrm{H}^{0}\left(E \otimes p_{1}^{*} \Omega_{\mathbb{P} m}^{p \vee} \otimes p_{2}^{*} \Omega_{\mathbb{P} n}^{q \vee}\right)$ and $g \in \mathrm{H}^{0}\left(E^{\vee} \otimes p_{1}^{*} \Omega_{\mathbb{P}^{m}}^{p} \otimes p_{2}^{*} \Omega_{\mathbb{P}^{n}}^{q}\right)$ with $\varphi(f)=s$ and $\psi(g)=s^{*}$. Then $g \circ f$ is an isomorphism by regarding as $f \in \operatorname{Hom}\left(p_{1}^{*} \Omega_{\mathbb{P} m}^{p} \otimes p_{2}^{*} \Omega_{\mathbb{P} n}^{q}, E\right)$ and $g \in \operatorname{Hom}\left(E, p_{1}^{*} \Omega_{\mathbb{P} m}^{p} \otimes p_{2}^{*} \Omega_{\mathbb{P n}}^{q}\right)$, which gives an inclusion from $p_{1}^{*} \Omega_{\mathbb{P}^{m}}^{p} \otimes p_{2}^{*} \Omega_{\mathbb{P}^{n}}^{q}$ to $E$ as a direct summand.

Example 2.2. Let us give an application of (2.1) to a vector bundle of $\mathbb{P}^{2} \times \mathbb{P}^{2}$. Let $E$ be an indecomposable vector bundle on $\mathbb{P}^{2} \times \mathbb{P}^{2}$. Then the following conditions are equivalent:

(a) $E \cong \Omega_{\mathbb{P}^{2}} \otimes \Omega_{\mathbb{P}^{2}}$

(b) $\mathrm{H}^{2}(E) \neq 0$ and $\mathrm{H}^{1}(E(1,1))=\mathrm{H}^{2}(E(0,1))=\mathrm{H}^{2}(E(1,0))=\mathrm{H}^{2}(E(-1,0))=$ $\mathrm{H}^{2}(E(0,-1))=\mathrm{H}^{3}(E(-1,-1))=0$.

\section{References}

[1] E. Arrondo and F. Malaspina, Cohomological characterization of vector bundles on Grassmannians of lines, J. Algebra 323 (2010), no. 4, 1098-1106.

[2] E. Ballico and F. Malaspina, Regularity and cohomological splitting conditions for vector bundles on multiprojective spaces, J. Algebra 345 (2011), 137 - 149. 
[3] M. C. Chang, Characterization of arithmetically Buchsbaum subschemes of codimension 2 in $\mathbb{P}^{n}$, J. Differential Geom. 31 (1990), 323-341.

[4] L. Costa and R. M. Miró-Roig, Cohomological characterization of vector bundles on multiprojective spaces, J. Algebra 294 (2005), 73-96, with a corrigendum in J. Algebra 319 (2008), 1336-1338.

[5] S. Goto, Maximal Buchsbaum modules over regular local rings and a structure theorem for generalized Cohen-Macaulay modules, ASPM 11(1987), 39-64.

[6] C. Miyazaki, A cohomological criterion for splitting of vector bundles on multiprojective space, Proc. Amer. Math. Soc. 143 (2015), 1435-1440.

[7] C. Miyazaki, Buchsbaum criterion of Segre products of vector bundles on multiprojective space, J. Algebra 467 (2016), 47-57.

[8] D. Mumford, Lectures on curves on an algebraic surface, Annals of Math. Studies 59 (1966), Princeton UP.

[9] J. Stückrad and W. Vogel, Buchsbaum rings and applications, Springer, 1986. 